\title{
Effects of $p 53$ Status of Tumor Cells and Combined Treatment With Mild Hyperthermia, Wortmannin or Caffeine on Recovery From Radiation-Induced Damage
}

\author{
Shin-ichiro Masunaga ${ }^{\text {a, f }}$, Keizo Tano ${ }^{\text {a }}$, Yu Sanada ${ }^{\text {a }}$, Minoru Suzuki ${ }^{b}$, \\ Akihisa Takahashic ${ }^{\mathrm{c}}$, Ken Ohnishi ${ }^{\mathrm{d}}, \mathrm{Koji}_{\mathrm{Ono}}^{\mathrm{e}}$
}

\begin{abstract}
Background: The aim of the study was to examine the dependency of $p 53$ status and the usefulness of mild hyperthermia (MHT) as an inhibitor of recovery from radiation-induced damage, referring to the response of quiescent $(\mathrm{Q})$ tumor cell population.

Methods: Human head and neck squamous cell carcinoma cells transfected with mutant TP53 (SAS/mp53) or with neo vector (SAS/neo) were injected subcutaneously into left hind legs of nude mice. Tumorbearing mice received 5-bromo-2'-deoxyuridine (BrdU) continuously to label all intratumor proliferating $(\mathrm{P})$ cells. They received high dose-rate $\gamma$-ray irradiation (HDR) immediately followed by localized MHT $\left(40{ }^{\circ} \mathrm{C}\right.$ for $2 \mathrm{~h}$ ), or caffeine or wortmannin administration, or low dose-rate $\gamma$-ray irradiation simultaneously with localized MHT or caffeine or wortmannin administration. Nine hours after the start of irradiation, the tumor cells were isolated and incubated with a cytokinesis blocker, and the micronucleus (MN) frequency in cells without BrdU labeling ( $=$ Q cells) was determined using immunofluorescence staining for BrdU.
\end{abstract}

Results: SAS/neo tumor cells, especially intratumor Q cell populations, showed a marked reduction in sensitivity due to the re-

Manuscript submitted May 13, 2019, accepted June 8, 2019

aParticle Radiation Biology, Institute for Integrated Radiation and Nuclear Science, Kyoto University, 2-1010, Asashiro-nishi, Kumatori-cho, Sennan-gun, Osaka 590-0494, Japan

bParticle Radiation Oncology, Institute for Integrated Radiation and Nuclear Science, Kyoto University, 2-1010, Asashiro-nishi, Kumatori-cho, Sennangun, Osaka 590-0494, Japan

${ }^{\circ}$ Gunma University Heavy Ion Medical Center, 3-39-22, Showa-machi, Maebashi, Gunma 371-8511, Japan

dDepartment of Biology, Center for Humanity and Sciences, Ibaraki Prefectural University of Health Sciences, 4669-2, Ami, Inashiki-gun, Ibaraki 3000394, Japan

eKansai BNCT Medical Center, Osaka Medical College, 2-7 Daigaku-machi Takatsuki, Osaka 569-8686, Japan

${ }^{f}$ Corresponding Author: Shin-ichiro Masunaga, Particle Radiation Biology, Division of Radiation Life Science, Institute for Integrated Radiation and Nuclear Science, Kyoto University, 2-1010, Asashiro-nishi, Kumatori-cho, Sennangun, Osaka 590-0494, Japan. Email: masunaga.shinichiro.6m@kyoto-u.jp

doi: https://doi.org/10.14740/wjon1203 covery from radiation-induced damage, compared with the total or Q tumor cells within SAS/mp53 tumors that showed little recovery capacity. The recovery from radiation-induced damage was thought to be a p53-dependent event. In both total and $\mathrm{Q}$ tumor cells within SAS/neo tumors, especially the latter, MHT efficiently suppressed the reduction in sensitivity caused by leaving an interval between HDR irradiation and the assay and decreasing the irradiation dose-rate, as well as the combination with wortmannin administration.

Conclusions: From the viewpoint of solid tumor control as a whole, including intratumor Q-cell control, non-toxic MHT is useful for suppressing the recovery from radiation-induced damage, as well as wortmannin treatment combined with $\gamma$-ray irradiation.

Keywords: Recovery from radiation-induced damage; Dose-rate effect; $p 53$ status; Mild hyperthermia; Wortmannin; Caffeine; Quiescent cell

\section{Introduction}

Hyperthermia is a heat treatment that directly targets cancer cells themselves or targets the environment surrounding tumor cells. In classical hyperthermic oncology, significant tumor cell killing is supposed to occur if cells or tissues are heated to over $42{ }^{\circ} \mathrm{C}$ for $1 \mathrm{~h}$ or more. Radio-sensitization and chemosensitization induced by heat treatment were speculated to be significant partly by inhibiting DNA damage repair [1]. However, clinical experience so far has taught us that we are unable routinely to achieve thermal dose goals of over $42{ }^{\circ} \mathrm{C}$ for 1 $\mathrm{h}$ or more. It is now known that cytotoxic temperatures are achieved only in small sub-volumes of tumors during typical hyperthermia treatments with currently available heating technologies (except with thermal ablation) [1]. The effects of hyperthermia at mild temperatures (MHT) $\left(39-41^{\circ} \mathrm{C}\right.$ for $\left.1-2 \mathrm{~h}\right)$ on tissues are subtle. However, the effects of MHT, including heat-mediated tumor reoxygenation and inhibition of sublethal and potentially lethal damage repair, provide a strong rationale for using MHT in combination with radiotherapy [2]. In addition, physiological and cellular effects of MHT can improve the delivery of drug vehicles, activate promoters for heat- 
mediated gene therapy and increase the immune response to tumors through a variety of mechanisms [1, 2].

Genomic instability is a major force driving human cancer development. The $p 53$ tumor suppressor gene serves a critical role in maintaining genomic stability during the cell cycle checkpoint in not only G1 but also the G2/M transition, as an effector of DNA repair and apoptosis. Wild-type $p 53$ is liable to activate apoptosis in response to DNA damage $[3,4]$. These actions of $p 53$ are potentially critical in determining the effectiveness of ionizing radiation. Actually, mutations in the $p 53$ tumor suppressor gene have been shown to have an impact on the clinical course of several cancers. Patients with cancers harboring p53 mutations often have a worse prognosis than those with tumors harboring wild-type $p 53[3,4]$. Thus, the genetic and functional status of the $p 53$ gene is thought to be an important factor in guiding therapeutic strategies for cancer patients.

Many cells in solid tumors are quiescent in situ but are still clonogenic [2]. These quiescent (Q) tumor cell populations have been thought to be more resistant to irradiation because of their much larger hypoxic fractions and greater potentially lethal damage repair (PLDR) capacities than proliferating $(\mathrm{P})$ tumor cells, mainly based on the characteristics of plateau-phase cultured cells in vitro $[5,6]$. Employing our method for selectively detecting the response of intratumor Q cell populations in vivo, we have already shown that all these characteristics could be applied to Q cells in solid tumors in vivo under conventional high dose-rate irradiation (HDR) conditions $[2,6]$. However, low dose-rate irradiation (LDR) was found to spare normal tissue from radiation-induced damage resulting in a greater therapeutic gain, because the therapeutic ratio is equal to the ratio of tumor control to normal tissue complications [7].

Two major pathways for the repair of potentially lethal DNA double-stranded breaks (dsbs) exist in mammalian cells. The non-homologous end-joining (NHEJ) pathway is imprecise, error-prone and mutagenic, and mutant cell lines lacking key components of this pathway all exhibit impaired kinetics of DNA dsb repair and exquisite radio-sensitivity. Homologous recombination (HR) is a more precise (errorfree) repair mechanism and is more important for the repair of dsbs in late-S and G2 when a sister chromatid is available for the recombination reaction. Cell lines with defects in HR also exhibit increased radio-sensitivity and decreased fidelity of repair $[3,4]$. Wortmannin is known to have the potential to hinder NHEJ repair by inhibiting a catalytic subunit of DNA-dependent protein kinase [8]. Caffeine is known to inhibit HR by targeting ataxia telangiectasia mutated protein kinase (ATM) and ATM- and Rad3-related protein kinase (ATR) [9].

Here, the usefulness of MHT, or wortmannin or caffeine treatment right after HDR or concurrently with LDR with low linear energy transfer (LET) radiation $\gamma$-rays, including the dependency on $p 53$ status of tumor cells using tumor cell lines with identical genetic backgrounds except for $p 53$ status, was evaluated in terms of the extent of the recovery from radiationinduced damage, using our method for selectively detecting the responses of the total $(=\mathrm{P}+\mathrm{Q})$ and $\mathrm{Q}$ tumor cell populations in solid tumors $[5,6]$.

\section{Materials and Methods}

\author{
Cells, tumors and mice
}

The human head and neck squamous cell carcinoma cell line SAS (provided by JCRB, Tokyo, Japan) was cultured at $37^{\circ} \mathrm{C}$ in Dulbeco's modified Eagle's medium (DMEM) containing 20 mM 2-[4-(2-hydroxyethyl)-1-piperazinyl]ethanesulfonic acid (HEPES) and $12.5 \%$ fetal bovine serum in a conventional humidified $5 \% \mathrm{CO}_{2}$ incubator. SAS cells show the phenotype of wild-type $p 53$ in radiation- and heat-induced signal transduction [4], although they have a point mutation at codon 336 of exon 10 in the $p 53$ gene [10]. The plasmids pC53-248, which contains an $m p 53$ gene (codon 248, from Arg to Trp) producing a dominant negative $m p 53$ protein, and pCMV-Neo-Bam, which contains a neo-resistant marker, were provided by B. Vogelstein (Johns Hopkins Oncology Center, Baltimore, MD, USA). These plasmids were linearized with HindIII. Confluent SAS cells, approximately $2 \times 10^{6}$ cells in a $75-\mathrm{cm}^{2}$ flask, were trypsinized, and the resulting cell suspension in phosphate-buffered saline (PBS) $(1 \mathrm{~mL})$ was transferred into an electroporation chamber. Cells were supplemented with linearized DNA $(10 \mu \mathrm{g} / 10 \mu \mathrm{L}$ of pC53-248 or pCMV-Neo-Bam), and electroporated three times at $600 \mathrm{~V}$. After standing for $30 \mathrm{~min}$ at room temperature, cells were plated onto dishes $10 \mathrm{~cm}$ in diameter in DMEM and incubated at $37^{\circ} \mathrm{C}$. Forty-eight hours later, cells were treated with G418 (geneticin, $200 \mu \mathrm{g} / \mathrm{mL}$, Sigma Chemical Co., St. Louis, MO, USA), an agent for the selection of transfected clones, and then incubated at $37^{\circ} \mathrm{C}$ for 14 days to allow colonies to form. Colonies resistant to G418 were isolated with cloning cylinders. Through these manipulations, two stable transfectants, SAS/ mp53 and SAS/neo, were established. SAS/neo cells have a functional wild-type $p 53$ protein, while SAS $/ m p 53$ cells express a dominant negative $p 53$ protein. The procedure used for transfection is described in detail elsewhere [11].

Cells were collected from exponentially growing cultures, and approximately $5.0 \times 10^{5}$ cells were inoculated subcutaneously into left hind legs of 6- to 7-week-old syngeneic female $\mathrm{Balb} / \mathrm{cA}$ nude mice. Three weeks after the inoculation, a tumor with a diameter of approximately $7 \mathrm{~mm}$ could be observed at each implanted site, whichever stable transfectant was used.

Mice were handled according to the Recommendations for Handling of Laboratory Animals for Biomedical Research, compiled by the Committee on Safety and Ethical Handling Regulations for Laboratory Animal Experiments, Kyoto University. All experimental procedures mentioned here were in accordance with institutional guidelines for the care and use of laboratory animals in research. Thus, Institutional Review Board approval was obtained. This study was conducted in compliance with all the applicable institutional ethical guidelines for the care, welfare and use of animals.

\section{Labeling with 5-bromo-2'-deoxyuridine (BrdU)}

Two weeks after the tumor cell inoculation, mini-osmotic pumps (Alzet model 2001, DURECT Corporation, Cupertino, 
CA, USA) containing BrdU dissolved in physiological saline $(250 \mathrm{mg} / \mathrm{mL})$ were implanted subcutaneously to label all $\mathrm{P}$ cells for 7 days. Administration of BrdU did not change the tumor growth rate. The tumors were approximately $7 \mathrm{~mm}$ in diameter on treatment. The labeling index (LI) after continuous labeling with $\mathrm{BrdU}$ was $48.4 \%$ (41.7-55.1\%) (mean $(95 \%$ confidence limit)) and $43.2 \%(37.0-49.4 \%)$ for SAS/neo and $\mathrm{SAS} / m p 53$ tumor cells, respectively, and reached a plateau level at these stages. Therefore, we regarded tumor cells not incorporating BrdU after continuous labeling as Q tumor cells.

\section{Treatment}

After the labeling with BrdU, tumor-bearing mice received whole body $\gamma$-ray irradiation. The $\gamma$-ray irradiation was performed with a cobalt-60 $\gamma$-ray irradiator at a dose-rate of 2.75 Gy/min as HDR with tumor-bearing mice held in a specially constructed device with the tail firmly fixed with an adhesive tape. Irradiated total doses were 4, 8.5, 14.5, 20 and 24 Gy. LDR was performed at a dose-rate of $0.035 \mathrm{~Gy} / \mathrm{min}(2.1 \mathrm{~Gy} / \mathrm{h})$ by maintaining an appropriate distance between the cobalt- 60 radiation source and the irradiated tumor-bearing mouse fixed within the specially constructed device. Irradiated total doses were 8.5, 14 and $21 \mathrm{~Gy}$. This dose-rate is almost the minimum value available at our institute without contaminating secondary $\gamma$-rays. According to ICRU Report 58 for radiotherapy for uterine cervical cancer, high, middle and low dose-rates are defined as $>0.2 \mathrm{~Gy} / \mathrm{min}(12 \mathrm{~Gy} / \mathrm{h}), 0.033-0.20 \mathrm{~Gy} / \mathrm{min}(2$ - $12 \mathrm{~Gy} / \mathrm{h})$ and $<0.033 \mathrm{~Gy} / \mathrm{min}(2 \mathrm{~Gy} / \mathrm{h})$, respectively [12]. Thus, if this definition is strictly applied, the reduced dose-rate employed corresponds to the middle dose-rate. However, in this study, because the employed reduced dose-rate was much smaller than the employed HDR, the term LDR is used to indicate the reduced dose-rate from here on.

Combined MHT $\left(40^{\circ} \mathrm{C}, 2 \mathrm{~h}\right)$ was performed right after HDR irradiation or started right before the beginning of LDR irradiation. Concerning MHT, the tumors grown in the left hind legs of mice were heated at $40^{\circ} \mathrm{C}$ by immersing the tumor-bearing foot in a waterbath. The mouse was held in a specially constructed device with the tail and right leg firmly fixed with adhesive tape. The left tumor-bearing leg was pulled down by a special sinker (approximately $45 \mathrm{~g}$ ) that was affixed to the skin of the toe with Superglue (Arone-arufa, Konishi, Osaka, Japan). The mice were then placed on a circulating waterbath maintained at the desired temperature. The mice were air-cooled during the heat treatment. Temperatures at the tumor center equilibrated within $3-4$ min after immersion in the waterbath and remained $0.2-0.3{ }^{\circ} \mathrm{C}$ below the bath's temperature. The waterbath's temperature was maintained at $0.3{ }^{\circ} \mathrm{C}$ above the desired tumor temperature [6].

In addition, wortmannin $(6 \mathrm{mg} / \mathrm{kg})$ or caffeine $(68.5 \mathrm{mg} /$ $\mathrm{kg}=1 / 2 \mathrm{LD}_{50}$ (mean lethal dose)) dissolved in physiological saline $[8,9]$ was also administered intraperitoneally immediately after HDR irradiation or immediately before the start of LDR irradiation. Each irradiation group also included mice that were not pretreated with BrdU.

In the HDR irradiation group, when a tumor approximately $7 \mathrm{~mm}$ in diameter was observed in the left leg of the mouse, irradiation was first performed, and wortmannin or caffeine was administered intraperitoneally to the mouse immediately thereafter. Alternatively, in some mice, tumors were treated with localized MHT instead of these drug administrations. In the LDR irradiation group, wortmannin or caffeine was first administered intraperitoneally to the mouse at the stage when a tumor approximately $7 \mathrm{~mm}$ in diameter was observed in the left leg of the mouse, and LDR irradiation was started immediately after that. Alternatively, instead of the administration of these drugs, some mice were treated with localized MHT for the tumor during the LDR irradiation. After the entire system including the equipment for localized MHT was assembled in the irradiation room and all preparations for heating the tumor implanted in the mouse were complete, the LDR irradiation is started simultaneously with the start of heating.

\section{Immunofluorescence staining of BrdU-labeled cells and micronucleus (MN) assay}

Nine hours after HDR or right after LDR with or without MHT, tumors were excised from the mice given BrdU, minced and trypsinized. Tumor cell suspensions thus obtained were incubated for $72 \mathrm{~h}$ in tissue culture dishes containing complete medium and $1.0 \mu \mathrm{g} / \mathrm{mL}$ of cytochalasin-B to inhibit cytokinesis while allowing nuclear division, and the cultures were then trypsinized and cell suspensions were fixed. After the centrifugation of fixed cell suspensions, the cell pellet was resuspended with cold Carnoy's fixative (ethanol/acetic acid $=3: 1$ in volume). The suspension was then placed on a glass microscope slide and the sample was dried at room temperature. The slides were treated with $2 \mathrm{M}$ hydrochloric acid for $60 \mathrm{~min}$ at room temperature to dissociate the histones and partially denature the DNA. The slides were then immersed in borax-borate buffer ( $\mathrm{pH} 8.5$ ) to neutralize the acid. BrdU-labeled tumor cells were detected by indirect immunofluorescence staining using monoclonal anti-BrdU antibody (Becton Dickinson, San Jose, CA, USA) and fluorescein isothiocyanate (FITC)-conjugated antimouse IgG antibody (Sigma, St. Louis, MO, USA). To observe the double staining of tumor cells with green-emitting FITC and red-emitting propidium iodide (PI), cells on the slides were treated with PI $(2 \mu \mathrm{g} / \mathrm{mL}$ in phosphate-buffered saline (PBS)) and monitored under a fluorescence microscope.

The MN frequency in cells not labeled with BrdU could be examined by counting the micronuclei in the binuclear cells that showed only red fluorescence. The MN frequency was defined as the ratio of the number of micronuclei in the binuclear cells to the total number of binuclear cells observed $[5,6]$.

The ratios obtained in tumors not pretreated with $\mathrm{BrdU}$ indicated the $\mathrm{MN}$ frequency at all phases in the total $(\mathrm{P}+\mathrm{Q})$ tumor cell population. More than 400 binuclear cells were counted to determine the MN frequency.

\section{Clonogenic cell survival assay}

The clonogenic cell survival assay was also performed in the mice given no BrdU using an in vivo-in vitro assay method. Tumors were disaggregated by stirring for $20 \mathrm{~min}$ at $37{ }^{\circ} \mathrm{C}$ 
Table 1. Plating Efficiencies and Micronucleus Frequencies at 0 Gy

\begin{tabular}{|c|c|c|}
\hline Treatment & SAS/neo & SAS/mp53 \\
\hline \multicolumn{3}{|l|}{ Plating efficiency (\%) } \\
\hline \multicolumn{3}{|l|}{ Total tumor cells } \\
\hline MHT & $43.2(39.3-47.1)$ & $20.9(18.0-23.8)$ \\
\hline Caffeine & $28.3(24.3-32.3)$ & $17.9(14.9-20.9)$ \\
\hline \multicolumn{3}{|l|}{ Micronucleus frequency } \\
\hline \multicolumn{3}{|l|}{ Total tumor cells } \\
\hline None & $0.038(0.032-0.044)$ & $0.072(0.064-0.080)$ \\
\hline MHT & $0.050(0.045-0.055)$ & $0.075(0.068-0.082)$ \\
\hline Caffeine & $0.088(0.079-0.097)$ & $0.137(0.127-0.147)$ \\
\hline MTH & $0.059(0.053-0.065)$ & $0.10(0.09-0.11)$ \\
\hline Caffeine & $0.114(0.105-0.122)$ & $0.177(0.164-0.190)$ \\
\hline Wortmannin & $0.116(0.103-0.129)$ & $0.181(0.161-0.201)$ \\
\hline
\end{tabular}

aNumbers in parentheses are 95\% confidence limits, determined using mean values, standard deviations and the numbers of observations on which the means and standard deviations were based. The difference between the values for SAS/neo and SAS/mp53 is statistically significant under each set of condition $(P<0.05)$. MHT: mild hyperthermia.

in PBS containing $0.05 \%$ trypsin and $0.02 \%$ ethylenediamine tetraacetic acid. The cell yield was $1.5(1.2-1.8) \times 10^{7} / \mathrm{g}$ and $3.4\left(2.6\right.$ - 4.2) $\times 10^{6} / \mathrm{g}$ for SAS/neo and SAS/mp53 tumors, respectively. Appropriate numbers of viable tumor cells from the single cell suspension were plated on 60 or $100 \mathrm{~mm}$ tissue culture dishes, and 16 days later, colonies were fixed with ethanol, stained with Giemsa, and counted. For the tumors that received no irradiation, the plating efficiencies for the total tumor cell populations and the $\mathrm{MN}$ frequencies for the total and Q tumor cell populations are shown in Table 1.

To confirm the stability of transfectants SAS/neo and SAS/ $m p 53$, parts of tumor cell suspensions obtained after $\gamma$-ray irradiation to solid tumors and tumor cells from part of the colonies grown using the in vivo-in vitro assay method were subjected to Western blotting for $p 53$ and Bax proteins as described in Ota et al [13]. Not only the protein level but also the function of $p 53$ can be detected because the bax gene is one of p53's targets. As a result, it was confirmed that the $p 53$ status of each transfectant was not changed by these experimental procedures. Three mice were used to assess each set of conditions and each experiment was repeated twice. To examine the differences between pairs of values, Student's $t$-test was used when variances of the two groups could be assumed to be equal; otherwise, the Welch $t$ test was used. P-values are from two-sided tests.

\section{Results}

Table 1 shows the plating efficiencies for the total tumor cell population and the $\mathrm{MN}$ frequencies without $\gamma$-ray radiation for the total and Q tumor cell populations in each tumor. Overall, SAS/mp53 tumor cells showed significantly higher surviving fraction (SF) in the total tumor cell populations and significantly higher $\mathrm{MN}$ frequencies in both the total and Q tumor cell populations $(\mathrm{P}<0.05)$. Further, $\mathrm{Q}$ tumor cells showed significantly higher $\mathrm{MN}$ frequencies than the total tumor cell population under each set of condition in each tumor $(\mathrm{P}<0.05)$. Wortmannin and caffeine induced significantly lower plating efficiencies and significantly higher $\mathrm{MN}$ frequencies in both the total and Q tumor cell populations within each tumor than absolutely no treatment $(\mathrm{P}<0.05)$. Furthermore, the values of the SF and the MN frequency for both drugs were almost the same. Thus, it follows that the dose of each drug employed here had an almost iso-effect in the absence of $\gamma$-ray irradiation. The combination with MHT caused slightly lower plating efficiency and slightly higher MN frequency in both populations than absolutely no treatment.

The clonogenic cell survival curves for total tumor cell populations and the net $\mathrm{MN}$ frequencies of total and $\mathrm{Q}$ tumor cell populations after $\gamma$-ray irradiation with HDR or LDR are shown in Figure 1a and b, respectively. For baseline correction, we used the net MN frequency to exclude the effect of MHT, wortmannin or caffeine in non-irradiated control tumors. The net $\mathrm{MN}$ frequency was the $\mathrm{MN}$ frequency in the irradiated tumors minus that in the non-irradiated tumors. On the whole, SAS/mp53 tumor cells and Q tumor cells were more radioresistant than $\mathrm{SAS} /$ neo tumor cells and the total tumor cells, respectively. The increase in the SF with the $9 \mathrm{~h}$ delayed 

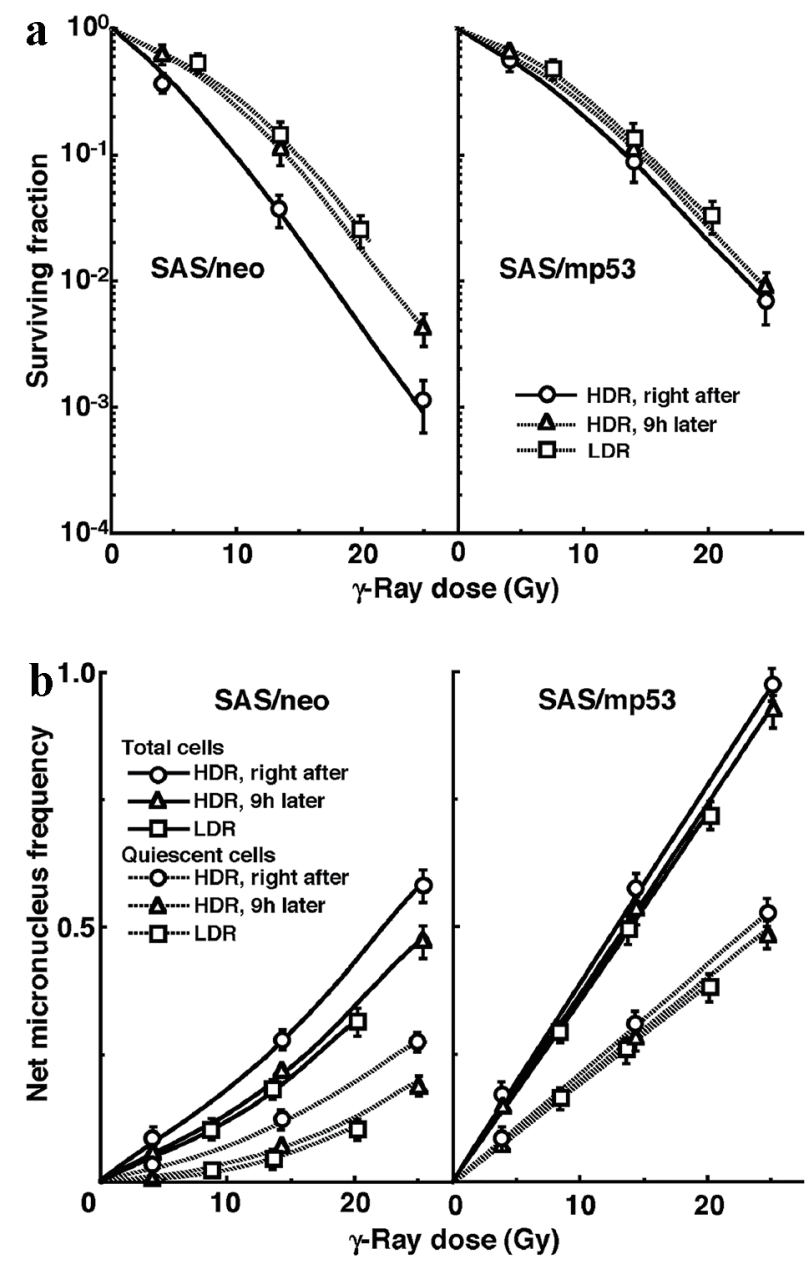

Figure 1. A marked reduction in sensitivity of SAS/neo tumor cells, especially quiescent tumor cells, compared with SAS/mp53 tumor cells with little recovery capacity. The clonogenic cell survival curves for total tumor cells and the net micronucleus frequencies for total and quiescent tumor cells immediately and $9 \mathrm{~h}$ after $\mathrm{y}$-ray irradiation with high dose-rate irradiation (HDR) and immediately after $\gamma$-ray irradiation with low dose-rate irradiation (LDR) are shown in (a) and (b), respectively. The left and right panels show SAS/neo and SAS/mp53 tumor cells, respectively. Bars represent $95 \%$ confidence limits. Three mice were used to assess each set of conditions and each experiment was repeated twice.

assay, that is, PLDR, and the increase in the SF after LDR were observed much more clearly in SAS/neo than in SAS/mp53. The decrease in the net MN frequency with the $9 \mathrm{~h}$ delayed assay and the decrease in the net MN frequency after LDR were more obvious in SAS/neo and Q cells than in SAS/mp53 and the total tumor cells, respectively. SAS/mp53 tumors showed little recovery capacity.

The clonogenic cell survival curves for total tumor cell populations and the net $\mathrm{MN}$ frequencies of total and Q tumor cell populations after $\gamma$-ray irradiation with HDR immediately followed by MHT, the administration of wortmannin or caffeine, or no treatment are shown in Figure 2a and b, respectively. PLDR was suppressed in combination with wortmannin much more clearly than with caffeine in both the total and Q
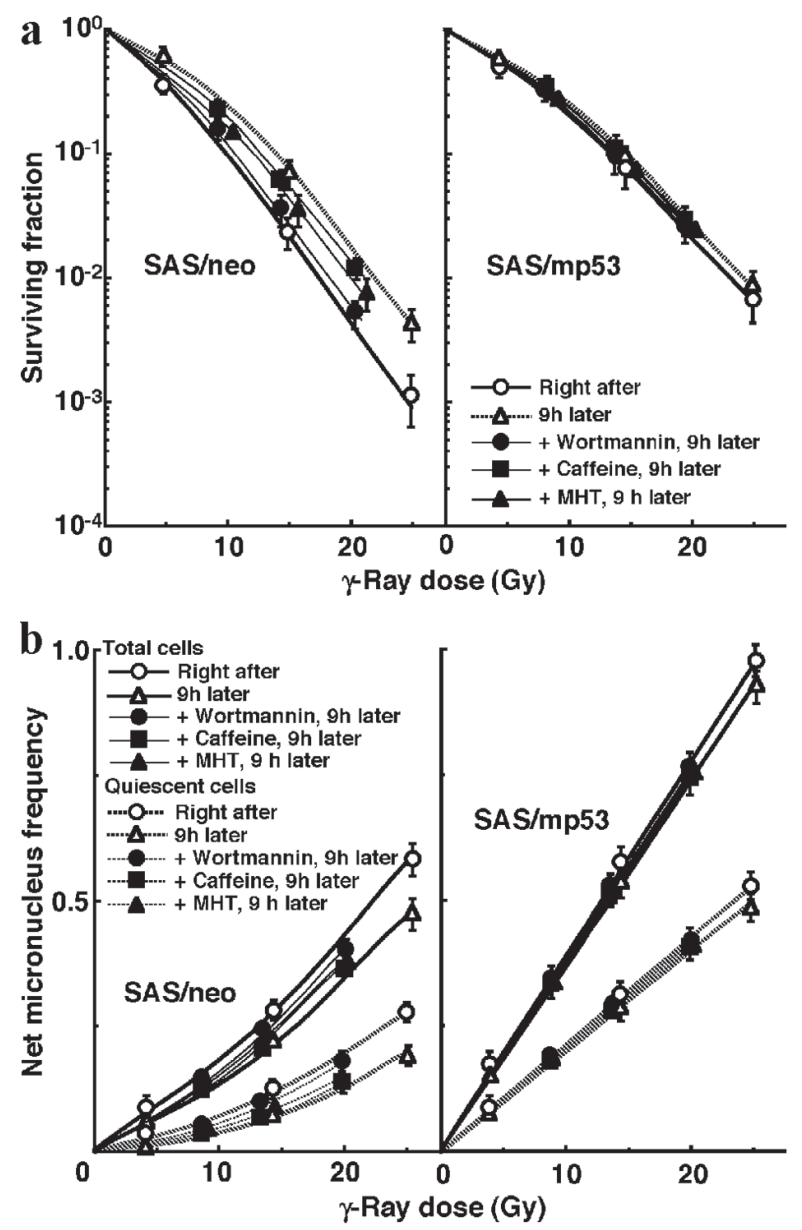

Figure 2. Mild hyperthermia efficiently suppressed the reduction in sensitivity caused by leaving an interval between high dose-rate irradiation (HDR) and the assay in both total and quiescent cells within SAS/neo tumors, as well as wortmannin administration. The clonogenic cell survival curves for total tumor cells and the net micronucleus frequencies for total and quiescent tumor cells immediately after and $9 \mathrm{~h}$ after $\mathrm{\gamma}$-ray irradiation with HDR immediately followed by mild hyperthermia, the administration of caffeine, that of wortmannin, or no treatment are shown in (a) and (b), respectively. The left and right panels show SAS/neo and SAS/mp53 tumor cells, respectively. Bars represent $95 \%$ confidence limits. Three mice were used to assess each set of conditions and each experiment was repeated twice.

tumor cells. MHT inhibited recovery from the damage induced by HDR irradiation more markedly than caffeine, although not as efficiently as wortmannin.

The clonogenic cell survival curves for total tumor cell populations and the net $\mathrm{MN}$ frequencies of total and $\mathrm{Q}$ tumor cell populations after $\gamma$-ray irradiation with LDR in simultaneous combination with MHT, wortmannin or caffeine, or no treatment are also shown in Figure $3 \mathrm{a}$ and b, respectively. The increase in the SF and the decrease in the net MN frequency after LDR were repressed in combination with wortmannin much more clearly than with caffeine in both total and Q tumor cell populations. The simultaneous combination with MHT also inhibited recovery from the damage induced during LDR irradiation more efficiently than the combination 

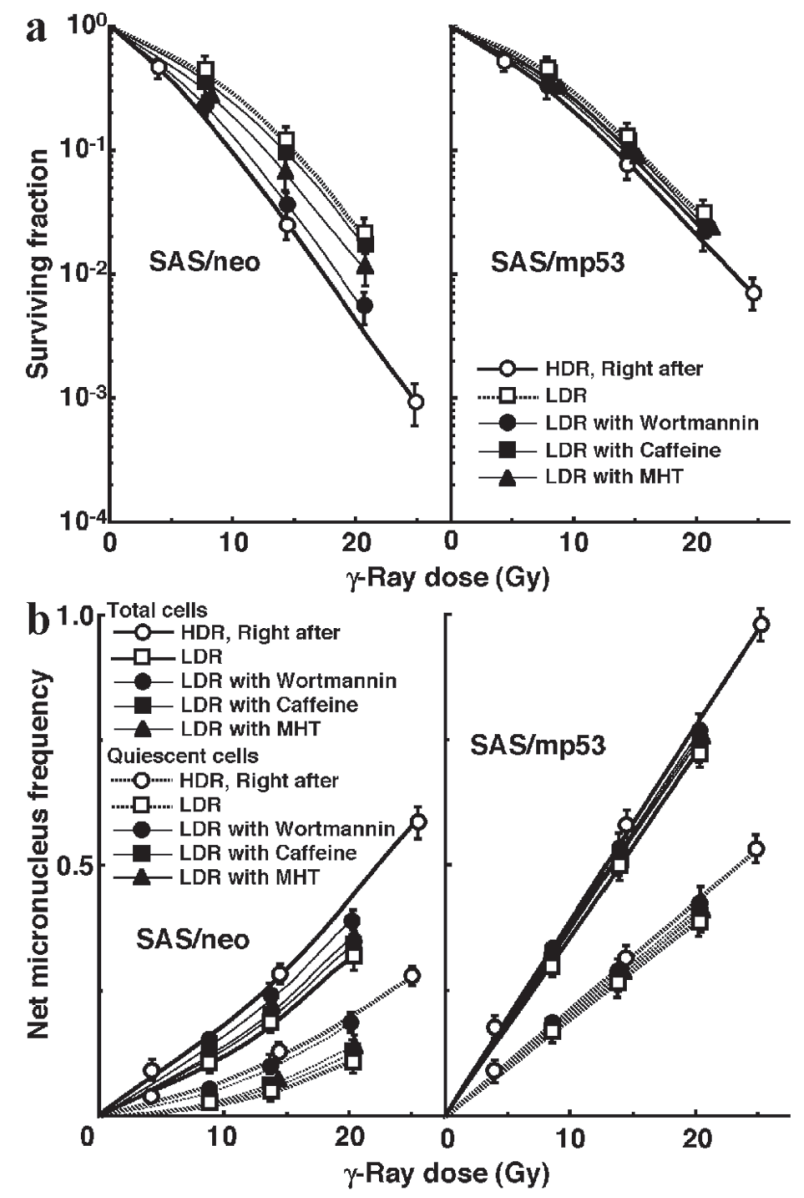

Figure 3. Mild hyperthermia efficiently suppressed the reduction in sensitivity caused by decreasing the irradiation dose rate in both total and quiescent cells within SAS/neo tumors, as well as wortmannin administration. The clonogenic cell survival curves for total tumor cell populations and the net micronucleus frequencies for total and quiescent cell populations immediately after $y$-ray irradiation with high dose-rate irradiation (HDR) and after $\mathrm{y}$-ray irradiation with low dose-rate irradiation (LDR) in simultaneous combination with mild temperature hyperthermia, the administration of caffeine or wortmannin, or no treatment are shown in (a) and (b), respectively. The left and right panels show SAS/neo and SAS/mp53 tumor cells, respectively. Bars represent $95 \%$ confidence limits. Three mice were used to assess each set of conditions and each experiment was repeated twice.

with caffeine, although again not as efficiently as wortman$\operatorname{nin}$.

To compare the cell survival curves between these two tumor cells, we calculated the dose ratios for SAS/mp 53 tumor cells relative to SAS/neo tumor cells. The factors were calculated by comparing the radiation doses to obtain SF of 0.04 in SAS/mp53 tumor cells with the doses required in $\mathrm{SAS} /$ neo tumor cells. The values of the dose ratios for SF of 0.04 were 1.35 (1.3 - 1.4), $1.05(1.0$ - 1.1), 1.2 (1.15 $1.25), 1.3(1.25-1.35)$ and $1.1(1.05-1.15)$ when tumors were excised from mice right after HDR, $9 \mathrm{~h}$ after HDR, $9 \mathrm{~h}$ after the beginning of MHT following HDR, $9 \mathrm{~h}$ after wortmannin administration following HDR and $9 \mathrm{~h}$ after caffeine administration following HDR, respectively. The values were
$1.05(1.0$ - 1.1), $1.1(1.05-1.15), 1.3(1.25-1.35)$ and 1.05 $(1.0-1.1)$ when tumors were excised from mice right after LDR, right after LDR combined with MHT, right after LDR combined with wortmannin and right after LDR combined with caffeine, respectively. The values were decreased by the delayed assay after LDR or a decreasing irradiation doserate because of the apparent recovery from radiation-induced damage in SAS/neo tumor cells, compared with SAS/mp53 tumor cells that showed little repair capacity. However, the clear recovery-inhibiting effect of wortmannin was kept almost constant. MHT showed more efficient recovery-inhibiting effect than caffeine, although again not as efficiently as wortmannin.

To evaluate the effect of wortmannin and caffeine on the recovery from damage induced by HDR or during LDR in total and Q tumor cell populations within these two tumors, dose-modifying factors were calculated in both tumor cell populations at various endpoints using the data given in Figures 1 through 3 (Table 2). Overall, regardless of the tumor cell populations, SAS/mp53 tumor cells showed little recovery capacity under any irradiation conditions. In SAS/neo tumor cells, whether $9 \mathrm{~h}$ after HDR or after LDR, the recovery capacities were significantly greater in $\mathrm{Q}$ tumor cell populations than total tumor cell populations $(\mathrm{P}<0.05)$. Wortmannin treatment right after HDR significantly inhibited recovery from the damage induced by HDR with a more remarkable inhibition in $\mathrm{Q}$ tumor cells than in the total tumor cell population. The combination of wortmannin and LDR significantly inhibited recovery from the damage caused by LDR more efficiently than did the combination of caffeine, with a more remarkable inhibition in Q tumor cells than in the total tumor cell population. MHT right after HDR irradiation significantly inhibited repair of the damage induced by HDR irradiation, with more marked inhibition in Q tumor cells than in the total tumor cell population, although not as efficiently as wortmannin treatment right after HDR irradiation. The simultaneous combination of MHT with LDR irradiation significantly inhibited recovery from the damage caused by LDR irradiation more efficiently than did the combination of caffeine, with more marked inhibition in Q tumor cells than in the total tumor cell population, although again not as efficiently as the wortmannin combination.

Table 3 shows the dose ratios of Q tumor cells relative to total tumor cell populations. These factors were used to compare the radiation doses necessary to obtain the net $\mathrm{MN}$ frequency of 0.1 in $\mathrm{Q}$ tumor cells with the doses required in the total tumor cell populations. All the values of the dose ratios were significantly larger than $1.0(\mathrm{P}<0.05)$. Wortmannin treatment right after HDR and simultaneously with LDR, especially in SAS/neo tumors, reduced the widened difference in $\gamma$-ray sensitivity between the total and Q tumor cell populations due to a greater recovery capacity in Q tumor cells than in the total tumor cell population at $9 \mathrm{~h}$ after HDR and after LDR, respectively. The application of MHT right after HDR irradiation and simultaneously combined with LDR irradiation reduced the widened difference in $\gamma$-ray sensitivity between the total and Q tumor cell populations due to greater recovery capacity in $\mathrm{Q}$ tumor cells than in the total tumor cell population $9 \mathrm{~h}$ after HDR irradiation and after LDR irradia- 
Table 2. Dose-Modifying Factors by Recoverya

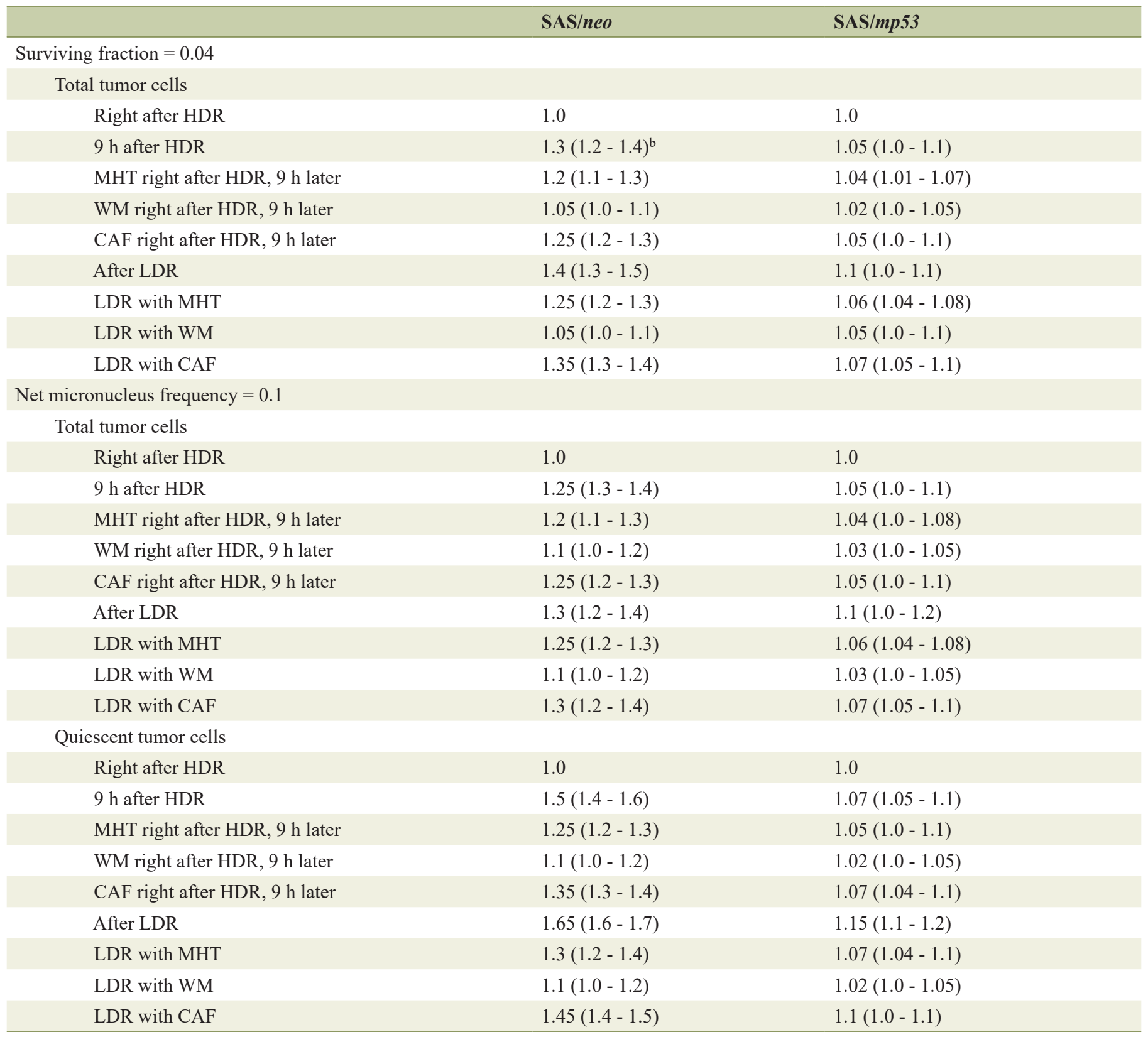

aThe dose of radiation required to obtain each endpoint with each treatment in relation to that required to obtain each endpoint immediately after HDR. bNumbers in parentheses are 95\% confidence limits, determined using mean values, standard deviations and the numbers of observations on which the means and standard deviations were based. Except for the conditions using treatment with wortmannin, the difference between the values for SAS/neo and SAS/mp53 is statistically significant under each set of condition $(P<0.05)$. HDR: high dose-rate irradiation; MHT: mild hyperthermia; WM: wortmannin; CAF: caffeine; LDR: low dose-rate irradiation.

tion, respectively, although again not as remarkably as wortmannin treatment.

\section{Discussion}

Potentially lethal damage (PLD) is the component of radiation damage that can be modified by post-irradiation conditions
[14]. Under ordinary circumstances, PLD causes cell death. Changing cellular growth conditions or the microenvironment of cells influences the expression of PLD or its repair (PLDR), and thereby influences sensitivity to radiation. PLDR is favored by conditions that maintain cells without encouraging or allowing them to divide. Conditions found in solid tumors, regions of which may be far from blood vessels and low in glucose and oxygen, have a low extracellular $\mathrm{pH}$, and show high 
Table 3. Dose Ratios ${ }^{a}$ for Quiescent Tumor Cells Relative to the Total Tumor Cell Population

\begin{tabular}{lll}
\hline & SAS/neo & SAS/mp53 \\
\hline Net micronucleus frequency $=0.1$ & & $1.9(1.8-2.0)$ \\
Right after HDR & $2.2(2.1-2.3)^{\mathrm{b}}$ & $1.95(1.85-2.05)$ \\
9 h after HDR & $2.35(2.25-2.45)$ & $1.9(1.8-2.0)$ \\
MHT right after HDR, 9 h later & $2.2(2.1-2.3)$ & $1.85(1.75-1.95)$ \\
WM right after HDR, 9 h later & $2.1(2.0-2.2)$ & $1.9(1.8-2.0)$ \\
CAF right after HDR, 9 h later & $2.3(2.2-2.4)$ & $1.95(1.85-2.05)$ \\
After LDR & $2.35(2.25-2.45)$ & $1.9(1.8-2.0)$ \\
LDR with MHT & $2.2(2.1-2.3)$ & $1.85(1.75-1.95)$ \\
LDR with WM & $2.1(2.0-2.2)$ & $1.9(1.8-2.0)$ \\
LDR with CAF & $2.3(2.2-2.4)$ & \\
\hline
\end{tabular}

aThe dose of radiation required to obtain a net micronucleus frequency of 0.1 in quiescent tumor cells in relation to that required to obtain a net micronucleus frequency of 0.1 in the total tumor cell population. ${ }^{b}$ Numbers in parentheses are $95 \%$ confidence limits, determined using mean values, standard deviations and the numbers of observations on which the means and standard deviations were based. The difference between the values for SAS/neo and SAS/mp53 is statistically significant under each set of condition ( $\mathrm{P}<0.05)$. HDR: high dose-rate irradiation; MHT: mild hyperthermia; WM: wortmannin; CAF: caffeine; LDR: low dose-rate irradiation.

concentrations of cellular waste products, may prevent cells from proliferating and thereby promote the repair of PLD. Extensive studies on PLDR suggest that DNA dsbs are potentially lethal lesions that can be converted into lethal damage [14]. It was recently reported that the conversion of potentially lethal lesions into lethal lesions might be a p53-dependent process and that PLDR was proportional to the percentage of radiationinduced DNA dsbs rejoined in $1 \mathrm{~h}$ in the cell lines with a normal p53 [4, 14].

Dose-rate is one of the principal factors determining the biologic consequences of a given absorbed dose. As the doserate is lowered and the exposure time extended, the biologic effect of a given dose is generally reduced. The dose-rate effect, which is very important in radiotherapy, results from the recovery from sublethal damage that occurs during a long radiation exposure [6]. Incidentally, sublethal damage repair is the operational term for the increase in cell survival that is observed if a given radiation dose is split into two fractions separated by a time interval. Because continuous LDR irradiation may be considered to be an infinite number of infinitely small fractions, the survival curve under these conditions also would be expected to have no shoulder and to be shallower than for a single acute exposure [7]. It was also reported that a normal functioning $p 53$ gene is indispensable for a repair of DNA damage induced under LDR irradiation [15, 16].

A cellular safeguard against genetic destabilization is activation of the $p 53$ tumor suppressor protein, which commonly responds to DNA damage signals by inducing apoptosis or regulating the cell cycle including DNA damage repair $[4,17]$. In fact, as also shown in our previous report [18] and this study, the net MN frequencies in SAS/neo tumor cells were lower than those in SAS/mp53 tumor cells under all conditions $(\mathrm{P}<0.05)$, probably resulting from exclusion of a higher number of radiation-induced apoptotic SAS/neo cells than SAS/mp53 cells.

Concerning whether PLDR after HDR or the repair during LDR, SAS/neo showed an apparent repair phenomenon in both total and Q tumor cell populations (Fig. 1, Table 2). No- tably, Q tumor cells in solid tumors with wild-type $p 53$ exhibited greater capacities of the recovery than the total tumor cell population, probably due to the intratumor conditions, that is, hypoxic, nutrition-depleted and low $\mathrm{pH}$ surroundings, where $\mathrm{Q}$ tumor cells came into existence $[1,2]$ (Fig. 1, Table 2). In contrast, no apparent recovery was observed in total or Q tumor cell populations within p53-mutated tumors (Fig. 1, Table 2).

It has been thought that decreasing the dose-rate reduces late effects in normal tissue much more than it decreases tumor control. Thus, the "therapeutic ratio" increases as the dose-rate decreases, because the therapeutic ratio is equal to the ratio of tumor control to normal tissue complications. Further, the difference between early and late effects for LDR radiotherapy, as well as improving the therapeutic ratio, allows the delivery of a complete treatment in a short time, allowing the effects of tumor repopulation to be minimized. In other words, decreasing the dose-rate increases the therapeutic ratio, limited by tumor cell repopulation $[7,14]$. This is the primary rationale for LDR radiotherapy. However, this rationale does not take into account the response of Q tumor cells. The current study showed that lowering the dose-rate decreases the effect on $\mathrm{Q}$ tumor cells more markedly than it reduces the effect on the total tumor cell population (Table 2). Therefore, considering the response of Q tumor cells, it follows that the therapeutic ratio does not always increase when the dose-rate is reduced. Thus, there is a need to overcome this disadvantage.

Wortmannin was originally described as a potent inhibitor of phosphoinositide 3-kinases (PI-3Ks), which are lipid kinases involved in insulin and other mitogenic signaling pathways. The kinase domain of PI-3K shares homology with ATM protein, which is a protein kinase that functions in DNA damageresponsive signaling pathways by phosphorylating target proteins. ATM is related to several other protein kinases involved in the regulation of eukaryotic cell cycle progression and DNA damage-triggered responses [8]. These proteins all contain a carboxy-terminal kinase domain that shares significant sequence homology with the kinase domains of mammalian and 
yeast PI-3Ks. In this family of PI-3K-related kinases, there is a catalytic subunit of the DNA-dependent protein kinase (DNAPKcs) that functions in the NHEJ DNA repair pathway. Thus, wortmannin shows the potential to inhibit the catalytic activities of DNA-PKcs, thereby hampering the NHEJ repair pathway $[4,8]$.

ATM and ATR are NHEJ-independent checkpoint regulators which facilitate repair by HR and are inhibited by caffeine $[4,9,19]$. Irradiation-induced DNA dsbs activate NHEJ and HR repair pathways and ATM- and ATR-dependent pathways that regulate checkpoint responses. Caffeine inhibits both the ATM- and ATR-regulated checkpoint responses which mainly affect HR [19]. Thus, caffeine is thought to preferentially inhibit HR repair.

The imprecise NHEJ pathway is the predominant repair process for cells in G0, G1, or early S-phase, and the precise HR pathway is more important for the repair of dsbs in late$\mathrm{S}$ and $\mathrm{G} 2[4,20]$. It was reported that wortmannin sensitized plateau-phase cultured cells, not $\mathrm{P}$ cells, to radiation in vitro [21]. Actually, in our study, Q tumor cells showed greater recovery capacity than the total tumor cell population in solid tumors with the wild-type $p 53$, and the recovery in both total and Q tumor cell populations was more effectively inhibited in combination with wortmannin than caffeine, in spite of an almost iso-effect induced by the drugs in the absence of $\gamma$-ray irradiation (Figs. 2 and 3, Tables 1 and 2). Further, it was also reported that the expression of RAD51, a critical mediator of $\mathrm{HR}$, is repressed by hypoxia in numerous cell lines, irrespective of $p 53$ status [3]. Taking into account that the intratumor Q cell population includes a much larger hypoxic fraction than the total tumor cell population, as shown in our previous report [6], the recovery in Q tumor cells in $p 53$-expressing solid tumors is thought to be predominantly due to the DNAPK-mediated NHEJ pathway. Anyway, wortmannin may be a promising candidate as a repair inhibitor in radiotherapy for solid tumors with wild-type $p 53$, if its toxicity is within the tolerance level for patients.

Loss-of-function of wild-type TP53 can result in loss of the G1/S cell-cycle checkpoint and an increase in HR. As $p 53$ seems to interact with RAD51, the absence of normal p53 function is thought to enhance RAD51-mediated DNA-pairing activity and HR, due to overexpression of RAD51 out of regulation by normal $p 53[4,17,20]$. Thus, HR is thought to be a principal mechanism of DNA dsb repair in SAS/mp53 cells. The very small recovery capacity of SAS/mp 53 tumor cells in vivo may show that the recovery in solid tumors with a mutant p53 is mainly due to, if anything, the NHEJ rather than HR.

The dose ratios for SAS/mp53 cells relative to $\mathrm{SAS} /$ neo cells showed that SAS/mp53 tumor cells within solid tumors are less radiosensitive than SAS/neo tumor cells. This is consistent with reports that tumor cells with a mutant $p 53$ gene were more radioresistant than those with a wild-type $p 53$ gene [18]. Since apparent recovery phenomena could be observed in solid tumors with a wild-type $p 53$ gene, the difference in sensitivity between $\mathrm{SAS} /$ neo and $\mathrm{SAS} / m p 53$ was slightly reduced without significant differences after recovery. However, the difference in sensitivity was almost constant with recoveryinhibiting wortmannin treatment.

MHT at $40-41^{\circ} \mathrm{C}$, if employed alone, is supposed to have almost no cytotoxic effects [1, 2]. Based on a formulation used extensively for measuring the thermal dose - the CEM $43^{\circ} \mathrm{CT} 90$ (number of cumulative equivalent minutes at $43{ }^{\circ} \mathrm{C}$ exceeded by $90 \%$ of the monitored points within the heated tumor) - the value is less than $1.0[1,2]$. Thus, almost no toxicity or cytocidal effect is supposed to be caused by MHT. However, both in vitro and in vivo, when combined simultaneously with LDR irradiation, MHT had been reported to inhibit efficiently cell recovery caused by the decrease in the irradiation dose-rate $[1,2,4]$. In the current study, the usefulness of MHT as a manipulation for suppressing PLDR and sublethal damage repair (SLDR) in both total and Q tumor cell populations was evaluated by applying MHT right after HDR irradiation and during LDR irradiation using $\gamma$-ray radiation, respectively, in terms of the responses of the total and Q tumor cell populations in solid tumors using our method for selectively detecting the response of Q tumor cell populations in vivo [6].

Solid tumors, especially human tumors, are thought to contain a high proportion of $\mathrm{Q}$ cells. The presence of these cells is probably due, in part, to a microregional deficiency in the concentrations of oxygen, glucose and other nutritional factors in the tumors caused by poor and heterogeneous tumor vascular supply [6]. This deficiency might promote MN formation in $\mathrm{Q}$ tumor cells at $0 \mathrm{~Gy}$ (Table 1) $[2,6]$. As shown here, $\mathrm{Q}$ tumor cells have lower radio-sensitivity than $\mathrm{P}$ tumor cells in solid tumors in vivo, irrespective of the $p 53$ status of tumor cells (Table 3) [18]. This means that more Q tumor cells survive after radiotherapy than $\mathrm{P}$ cells. Consequently, the control of Q tumor cells also has a great impact on the outcome of radiation therapy. Thus, from the viewpoint of solid tumor control as a whole, including intratumor Q-cell control, nontoxic MHT is thought to be useful for suppressing the recovery from radiation-induced damage, as well as wortmannin treatment combined with $\gamma$-ray irradiation.

\section{Acknowledgments}

The first author really appreciates the very kind donation of SAS/neo and SAS/mp53 tumor cells from Dr. Takahashi, Ph.D. and Dr. Ohnishi, Ph.D.

\section{Financial Disclosure}

This study was supported in part by a grant-in-aid for Scientific Research (B) (15H04295) and (C) (19K08171) from the Japan Society for the Promotion of Science.

\section{Conflict of Interest}

The authors have no conflict of interest.

\section{Informed Consent}

Informed consent was not obtained, because this study was not 
related to a clinical but a basic research using laboratory animals.

\section{Author Contributions}

Keizo Tano, Yu Sanada and Minoru Suzuki helped in performing the animal experiment. Akihisa Takahashi and Ken Ohnishi donated tumor cell lines with identical genetic backgrounds except for $p 53$ status and confirmed the stability of transfectants $\mathrm{SAS} /$ neo and SAS/mp53. Koji Ono gave support in analyzing the data. All authors read and approved the final manuscript.

\section{References}

1. Masunaga S. Tumor microenvironment and hyperthermia. In: Kokura S, Yoshikawa T, Ohnishi T, eds. Hyperthermic oncology from bench to bedside. Singapore: Springer, 2016; p. 151-169.

2. Vaupel PW, Kelleher DK. Pathophysiological and vascular characteristics of tumours and their importance for hyperthermia: heterogeneity is the key issue. Int J Hyperthermia. 2010;26(3):211-223.

3. Horn HF, Vousden KH. Coping with stress: multiple ways to activate p53. Oncogene. 2007;26(9):1306-1316.

4. Ohnishi T. Cellular responses in signal transduction pathways induced by hyperthermia. In: Kokura S, Yoshikawa T, Ohnishi T, eds. Hyperthermic oncology from bench to bedside. Singapore: Springer, 2016; p. 49-60.

5. Masunaga S, Ono K, Abe M. A method for the selective measurement of the radiosensitivity of quiescent cells in solid tumors - combination of immunofluorescence staining to BrdU and micronucleus assay. Radiat Res. 1991;125(3):243-247.

6. Masunaga S, Nishimura Y, Hiraoka M, Abe M, Takahashi M, Ono K. Efficacy of mild temperature hyperthermia in combined treatments for cancer therapy. Thermal Medicine. 2007;23(3):103-112.

7. Van der Kogel AJ, Joiner MC. The dose-rate effect. In: Joiner MC, van der Kogel AJ, eds. Basic Clinical Radiobiology. 5th ed. Boca Raton: CRC Press, 2019:143-151.

8. Hashimoto M, Rao S, Tokuno O, Yamamoto K, Takata M, Takeda S, Utsumi H. DNA-PK: the major target for wortmannin-mediated radiosensitization by the inhibition of DSB repair via NHEJ pathway. J Radiat Res. 2003;44(2):151-159.

9. Bohm L, Roos WP, Serafin AM. Inhibition of DNA repair by Pentoxifylline and related methylxanthine derivatives. Toxicology. 2003;193(1-2):153-160.

10. Jia LQ, Osada M, Ishioka C, Gamo M, Ikawa S, Suzuki
$\mathrm{T}$, Shimodaira H, et al. Screening the p53 status of human cell lines using a yeast functional assay. Mol Carcinog. 1997;19(4):243-253.

11. Ohnishi K, Wang X, Takahashi A, Ohnishi T. Contribution of protein kinase $\mathrm{C}$ to p53-dependent WAF1 induction pathway after heat treatment in human glioblastoma cell lines. Exp Cell Res. 1998;238(2):399-406.

12. International Commission on Radiation Units and Measurements. ICRU Report 58: Dose and volume specification for reporting interstitial therapy. Bethesda: ICRU. 1997.

13. Ota I, Ohnishi K, Takahashi A, Yane K, Kanata H, Miyahara H, Ohnishi T, et al. Transfection with mutant p53 gene inhibits heat-induced apoptosis in a head and neck cell line of human squamous cell carcinoma. Int J Radiat Oncol Biol Phys. 2000;47(2):495-501.

14. Hall EL, Giaccia AJ. Fractionated radiation and the doserate effect. In: Hall EJ, Giaccia AJ, eds. Radiobiology for the Radiologist, 7th ed. Philadelphia: Lippincott Williams \& Wilkins, 2012, 67-85.

15. Kato F, Ootsuyama A, Nomoto S, Kondo S, Norimura T. Threshold effect for teratogenic risk of radiation depends on dose-rate and p53-dependent apoptosis. Int J Radiat Biol. 2001;77(1):13-19.

16. Coleman MA, Yin E, Peterson LE, Nelson D, Sorensen $\mathrm{K}$, Tucker JD, Wyrobek AJ. Low-dose irradiation alters the transcript profiles of human lymphoblastoid cells including genes associated with cytogenetic radioadaptive response. Radiat Res. 2005;164(4 Pt 1):369-382.

17. Takahashi A. Inhibition of DNA repair system activity. In Kokura S, Yoshikawa T, Ohnishi T, eds. Hyperthermic Oncology from Bench to Bedside. Singapore: Springer, 2016; p. 91-108.

18. Masunaga S, Ono K, Takahashi A, Ohnishi T, Kinashi Y, Takagaki M. Radiobiological characteristics of solid tumours depending on the p53 status of the tumour cells, with emphasis on the response of intratumour quiescent cells. Eur J Cancer. 2002;38(5):718-727.

19. Wang H, Wang X, Iliakis G, Wang Y. Caffeine could not efficiently sensitize homologous recombination repairdeficient cells to ionizing radiation-induced killing. Radiat Res. 2003;159(3):420-425.

20. Takahashi A. Molecular damage: hyperthermia alone. In: Kokura S, Yoshikawa T, Ohnishi T, eds. Hyperthermic Oncology from Bench to Bedside. Singapore: Springer, 2016; p. 19-32.

21. Kubota N, Ozawa F, Okada S, Inada T, Komatsu K, Okayasu R. The phosphatidylinositol 3-kinase inhibitor wortmannin sensitizes quiescent but not proliferating MG-63 human osteosarcoma cells to radiation. Cancer Lett. 1998;133(2):161-167. 